\title{
Human Atrial Cell Models to Analyse the Effect of Extracellular Calcium on Action Potential Duration
}

\author{
Fazeelat Mazhar ${ }^{1}$, Chiara Bartolucci ${ }^{1}$, Stefano Severi $^{1}$ \\ ${ }^{1}$ University of Bologna, Cesena, Italy
}

\begin{abstract}
Regulation of cardiac electrical and mechanical activities are highly dependent on L-type calcium current $\left(I_{C a L}\right)$. Moreover, the phenomenon of $I_{\text {CaL }}$ inactivation plays a crucial role in adjusting action potential repolarization. This paper investigates the relationship between extracellular calcium concentration, $\left[\mathrm{Ca}^{+2}\right]_{0}$, and action potential (AP). In particular, calcium-dependent inactivation (CDI) of $I_{C a L}$ has been analyzed to quantify its dependence on $\left[\mathrm{Ca}^{+2}\right]_{O}$ and its impact on AP duration.

We have analyzed three human atrial cell models (hAM) by testing benchmarks like AP, $I_{C a L}$, and $I_{N C X}$ (sodiumcalcium exchange current) with the variation of $\left[\mathrm{Ca}^{+2}\right]_{O}$. The role of CDI was assessed by simulating the AP-clamp technique followed by 1) running the baseline model under normal CDI mechanism and 2) running the model without the CDI mechanism.

The comparison of three hAMs shows that besides the contribution of CDI, driving force $(D F)$, and $I_{N C X}$ current should also be considered while studying the relationship between AP duration (APD) and $\left[\mathrm{Ca}^{+2}\right]_{0}$. None of the hAMs reproduced in a physiologically correct way the effects of $\left[\mathrm{Ca}^{+2}\right]_{0}$ on APD, therefore our results call for a new hAM able to reproduce correctly the $[\mathrm{Ca}+2]$ o effect
\end{abstract}

\section{Introduction}

Atrial Fibrillation (AF) is caused by abnormal heart rhythm leading to disorganized and rapid activities of cardiac atria [1]. The AF-induced contractile dysfunction is mainly associated with the ionic current remodeling process. This electrical remodeling results in a shortened action potential duration (APD) and effective refractory period $[2,3]$.

Cardiac modeling represents an efficient tool to investigate the ionic mechanisms involved at the cell level and providing useful insights into different atrial pathologies, including AF, especially when experimental data are lacking.

One of the key contributions to APD shortening can be associated with disordered $\mathrm{Ca}^{+2}$ handling. Many studies have been conducted to elucidate the effect of variations in intracellular calcium concentration [4-6] but the effect of extracellular $\mathrm{Ca}^{+2}$ concentration $\left[\mathrm{Ca}^{+2}\right]_{\mathrm{O}}$ has not yet been fully understood.

Many different human atrial AP models (hAMs) are currently available and could help the investigation; generally, all were validated only with the standard electrolyte concentrations used in experiments and it happens that they did not reproduce the correct relationship between APD and $\left[\mathrm{Ca}^{+2}\right]_{\text {o. }}$.

The experimental data on the atrial APD- $\left[\mathrm{Ca}^{+2}\right]_{0}$ dependence, are very poor: from our knowledge, the only one could be found in [7] and is reported in Figure 1, where it is shown that by reducing $\left[\mathrm{Ca}^{+2}\right]_{\mathrm{O}}$ the APD is increasing.

L-type calcium current $\left(\mathrm{I}_{\mathrm{CaL}}\right)$ is the ionic current most affected by the extracellular $\mathrm{Ca}^{2+}$ changes and, therefore, affecting the APD. Two mechanisms have been hypothesized: i) an increased $\left[\mathrm{Ca}^{+2}\right]_{\mathrm{O}}$ leads to elevated driving force (DF, i.e., the difference between membrane potential $\mathrm{V}_{\mathrm{m}}$ and equilibrium potential $\mathrm{E}_{\mathrm{Ca}}$ ) causing an increased $\mathrm{I}_{\mathrm{CaL}}$ hence a prolonged APD; ii) the calciumdependent inactivation (CDI) gets strengthened resulting in a reduced APD. For this reason, while analyzing the relationship between APD- $\left[\mathrm{Ca}^{+2}\right]_{0}$ both factors (driving force and CDI) should be equally considered. The aim of this paper is 1) to study the response to $\left[\mathrm{Ca}^{+2}\right]_{\mathrm{O}}$ changes on APD in three hAMs: Courtemanche et al. [8], Koivumaki et al. [6], and Ellinwood et al. [9]; 2) to dissect by using AP-clamp technique and quantify the role of the different mechanisms involved: i) $I_{C a L} \mathrm{CDI}$ dependence on $\left[\mathrm{Ca}^{+2}\right]$, ii) the dependence of $\mathrm{I}_{\mathrm{CaL}}$ driving force on $\left[\mathrm{Ca}^{+2}\right]_{\mathrm{o}}$, iii) $\left[\mathrm{Ca}^{+2}\right]_{\mathrm{O}}$ dependence of $\mathrm{I}_{\mathrm{NCX}}$ ionic.

\section{Methods}

We have used three different hAMs: Courtemanche et al. [8] Koivumaki et al. [6], and Ellinwood et al. [9] were referred to as CRN, KM, and EM in the paper. Using the three hAMs the effect of $\left[\mathrm{Ca}^{+2}\right]_{\mathrm{O}}$ was analyzed by varying it among the values of [ $\left.\begin{array}{ll}0.9 & 1.82 .7\end{array}\right](\mathrm{mM})$. With this set of values, firstly the original behavior of the hAMs was analyzed.

Secondly, the models were modified to test the CDI dependence on varying $\left[\mathrm{Ca}^{+2}\right]_{\mathrm{o}}$. This was carried out by using the AP-clamp [10] technique instead of the conventional voltage clamp. AP-clamp explores the contribution of each ionic current in response to a natural 
AP waveform. The technique proceeds with a native AP waveform recorded at a steady-state (600s) and $\left[\mathrm{Ca}^{+2}\right]_{\mathrm{O}}=1.8 \mathrm{mM}$ used as a stimulus for the models instead of any rectangular or ramp voltage waveform. The state variables' values were recorded for each $\left[\mathrm{Ca}^{+2}\right]_{0}$ concentration at steady state. These values were used as initial conditions for the AP-clamp experiments. All the experiments were carried out at a constant rate of basic cycle length $(\mathrm{BCL})=1000 \mathrm{~ms}$. For the noCDI simulations, the $\mathrm{Ca}^{+2}$ dependent gate was turned off in the $\mathrm{I}_{\mathrm{CaL}}$ equation and the experiment was repeated for the same set of concentrations.

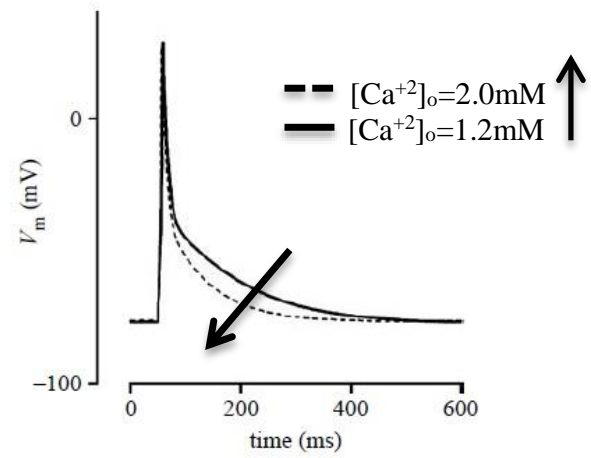

Figure 1. Superimposed AP recordings from human atrial myocyte superfused with $1.2 \mathrm{mM}$ (solid curve) and $2.0 \mathrm{mM}$ (dashed curve), adapted from [7].

The third analysis was a modification of the DF in the EM model, by making it independent of $\mathrm{Ca}$ concentrations. In particular, the concetrations involved are the sarcolemma (SL) calcium (SL space in most of the models is subdivided into junctional $\left[\mathrm{Ca}^{+2}\right]_{\mathrm{j}}$ and sub-sarcolemma compartments $\left[\mathrm{Ca}^{+2}\right]_{\mathrm{sl}}$ ) and $\left[\mathrm{Ca}^{2+}\right]_{\mathrm{o}}$. By fixing the SL Ca concentrations to their diastolic values $\left(\left[\mathrm{Ca}^{+2}\right]_{\mathrm{j}}=0.34 \mu \mathrm{M}\right.$ and $\left.\left[\mathrm{Ca}^{+2}\right]_{\mathrm{sl}}=0.24 \mu \mathrm{M}\right)$ and $\left[\mathrm{Ca}^{2+}\right]_{\mathrm{o}}=1.8 \mathrm{mM}$, the trend of $\mathrm{I}_{\mathrm{CaL}}$ was analyzed in CDI condition using AP-clamp.

All the hAMs are based on the set of ordinary differential equations depicting a dynamic process with a set of state variables. The differentiation formulas were solved using a variable order solver (ode15s) implemented in Matlab (Mathworks Inc., Natick, MA, USA). APD 90 was calculated at $90 \%$ repolarization of AP after $600^{\text {th }}$ beat. The current integrals were obtained by applying the trapz Matlab function over the time interval corresponding to $\mathrm{APD}_{90}$.

\section{Results}

The choice of an appropriate range of $\left[\mathrm{Ca}^{2+}\right]_{\mathrm{o}}$ should be consistent with the in vitro experimental data on which the model is based on [7]. Figure 1 depicts the human atrial action potential curves based on the experimental recordings for $\left[\mathrm{Ca}^{2+}\right]_{0}=1.2$ and $2.0 \mathrm{mM}$. In our simulations, the value of $\left[\mathrm{Ca}^{2+}\right]_{O}$ was set $1.8 \mathrm{mM}$ as a standard for running control models under current clamp setting. The variation of $\left[\mathrm{Ca}^{2+}\right]_{0}$ is a main contributor to APD variations. A rise in $\left[\mathrm{Ca}^{2+}\right]_{\mathrm{o}}$ results in an increased influx of $\mathrm{Ca}$ ions hence an increased $\mathrm{I}_{\mathrm{CaL}}$ which prolongs the APD. This gives rise to the CDI negative feedback that reduces $\mathrm{I}_{\mathrm{CaL}}$ current, therefore, shortening the APD. The overall hypothesized effect is the inverse relation between APD and $\left[\mathrm{Ca}^{2+}\right]_{\mathrm{o}}$ suggested by Figure 1 .

Figure 2 shows the three hAMs that were run at three different $\left[\mathrm{Ca}^{2+}\right]_{\mathrm{o}}$ values. The graph depicts action potential $\mathrm{Vm}, \mathrm{I}_{\mathrm{CaL}}$, and $\mathrm{I}_{\mathrm{NCX}}$ trends in steady state. CRN and $\mathrm{KM}$ models have similar behavior in terms of APD i.e., inverse relation to changing $\left[\mathrm{Ca}^{2+}\right]_{\mathrm{o}}$. This trend is quantified in
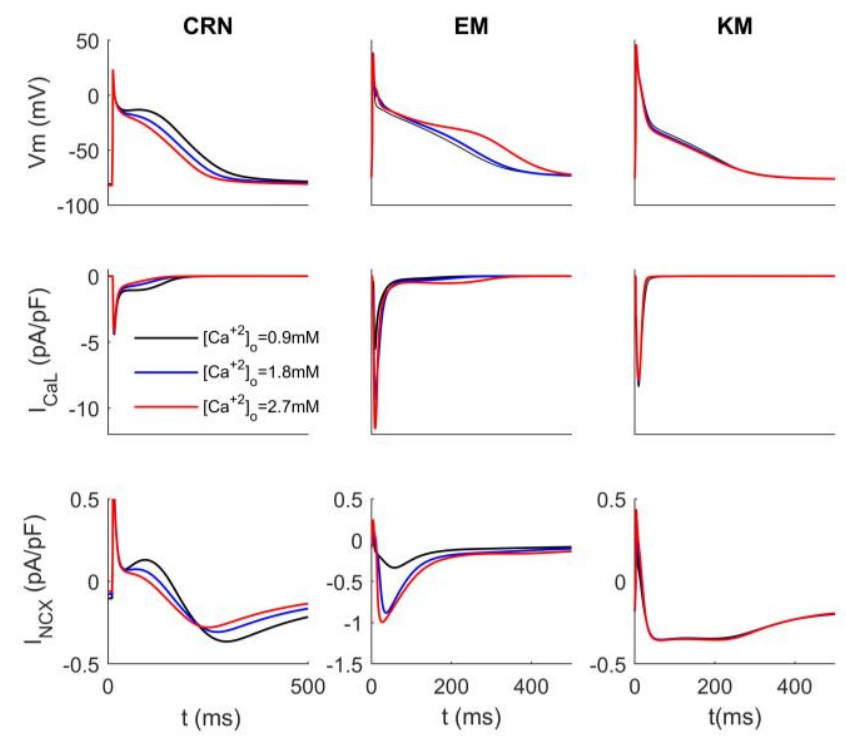

Figure 2: Comparison of AP (top), $\mathrm{I}_{\mathrm{CaL}}$ (middle) and $\mathrm{I}_{\mathrm{NCX}}$ (bottom) obtained with three hAMs (CRN, EM and KM) baseline codes for $\left[\mathrm{Ca}^{+2}\right]_{\mathrm{o}}=[0.9$ (black), 1.8 (blue), 2.7(red) $]$ at steady state $(600 \mathrm{~s}$ and BCL=1000ms). 
Figure 3. The trend is the same for both models with a clear difference of slope between the two (in CRN the slope is more accentuate) while the EM model shows a largely reverse relation.

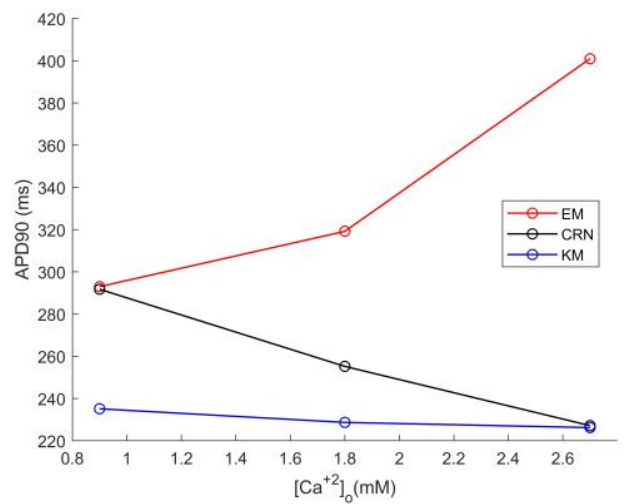

Figure 3: $\mathrm{APD}_{90}$ response to $\left[\mathrm{Ca}^{+2}\right]_{\mathrm{o}}$ variations on all three hAMs (EM, CRN and KM).

The quantification of the CDI mechanism was carried out using the AP-clamp technique where currents were analyzed for two different scenarios: for both CDI+VDI mechanism and for VDI only where calcium-dependent gates were turned off. Figure 4 shows current traces for all three hAMs, where CRN and KM are behaving in a similar fashion. In both hAMs, under AP-clamp, $\left[\mathrm{Ca}^{2+}\right]_{\mathrm{o}}$ variations has only a slight influence on $\mathrm{I}_{\mathrm{CaL}}$, which becomes completely unsensitive to $\left[\mathrm{Ca}^{2+}\right]_{\mathrm{o}}$ when CDI is turned off. This trend can be appreciated in Figure 5, where integration of $\mathrm{I}_{\mathrm{CaL}}$ under AP has been plotted. Here both hAMs show slight slope under CDI+VDI and completely flat curves under VDI only. On the other hand, the CDI mechanism has a strong impact on the EM model, dramatically changing how the amount of charge carried
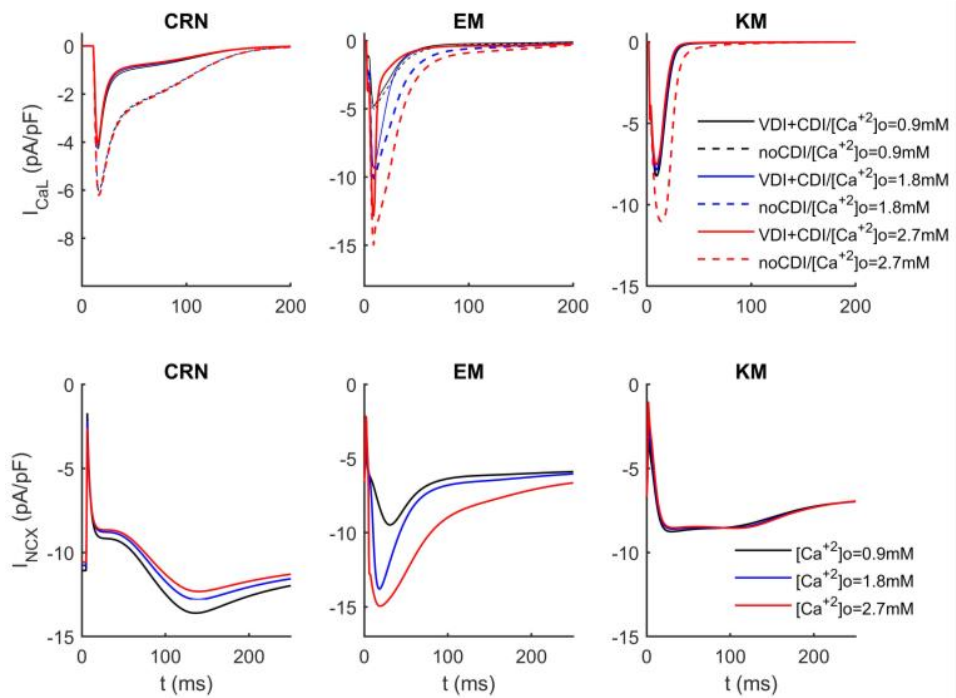

Figure 4: Comparison of $\mathrm{I}_{\mathrm{CaL}}$ (top) and $\mathrm{I}_{\mathrm{NCX}}$ traces for all three hAMs (CRN, EM and KM) using AP-clamp technique. $\mathrm{I}_{\mathrm{CaL}}$ has been measured for both VDI+CDI (solid lines) and NoCDI (dashed lines) conditions at different $\left[\mathrm{Ca}{ }^{2+}\right]_{\mathrm{o}} \cdot \mathrm{I}_{\mathrm{NCX}}$ has been evaluated for VDI+CDI only. by $\mathrm{I}_{\mathrm{CaL}}$ depends on $\left[\mathrm{Ca}^{+2}\right]_{\mathrm{o}}$ (compare continuous and dashed red lines in Figure 5). Nevertheless, the EM model still not correctly reproduces the APD- $\left[\mathrm{Ca}^{+2}\right]_{\mathrm{o}}$ relation (Figure 3, blue line). To better understand the cause, we made the DF independent of $\mathrm{Ca}^{+2}$ concentrations (see

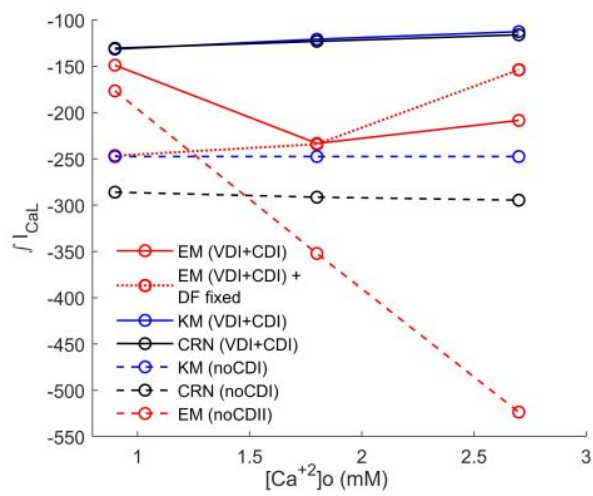

Figure 5: Integration of $I_{C a L}$ current under $A P$ for all three hAMs for CDI+VDI and noCDI (for EM model also with and without fixed DF).

Methods). In this configuration the EM model shows an opposite behavior (Figure 5, dotted red line) proving that CDI is effective in decreasing the current upon increase of $\left[\mathrm{Ca}^{+2}\right]_{\mathrm{o}}$, however this is overcome by the opposing effect of $\left[\mathrm{Ca}^{+2}\right]_{0}$ modulation of $\mathrm{DF}$, leading to the resulting $\mathrm{AP}$ prolongation.

We also analysed the importance of $\mathrm{I}_{\mathrm{NCX}}$ in this phenomenon, as shown in Figure 4 (bottom panel) and Figure 6. Similar trend of KM and CRN models, with a small difference in the slope value, was observed, whereas EM shows a significant dependence on $\left[\mathrm{Ca}^{+2}\right]_{\mathrm{o}}$ : if $\left[\mathrm{Ca}^{2+}\right]_{\mathrm{o}}$ increases, the inward $\mathrm{I}_{\mathrm{NaCa}}$ indeed increases, and the APD will prolong as a consequence. 


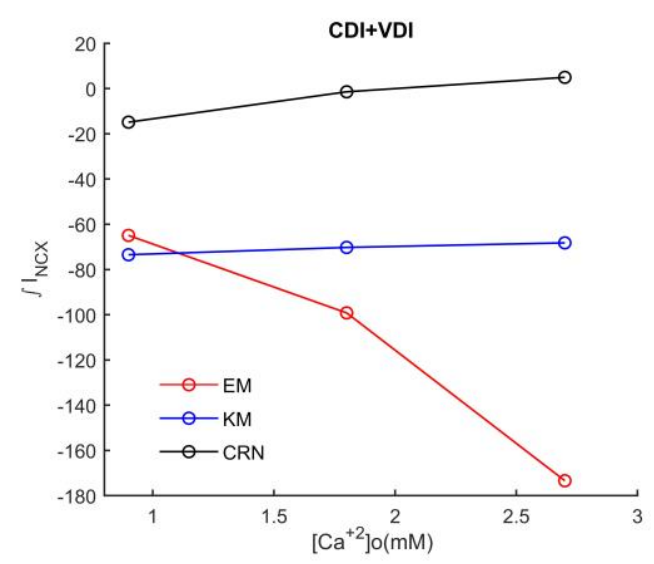

Figure 6: Integration of $\mathrm{I}_{\mathrm{NCX}}$ current under AP for all three hAMs.

\section{Discussion and Conclusions}

Our analysis suggests that among the analyzed hAMs CRN and KM qualitatively reproduce the real behavior of cells when $\left[\mathrm{Ca}^{+2}\right]_{0}$ is varied whereas EM does not (Figure 1 vs Figure 2). The current mostly contributing to the overall APD trend is $\mathrm{I}_{\mathrm{CaL}}$, but $\left[\mathrm{Ca}^{+2}\right]_{\mathrm{o}}$ influences $\mathrm{I}_{\mathrm{CaL}}$ in two opposing ways by modulating DF and CDI. In silico analysis allowed us to precisely quantify these contributions in terms of changes in the amount of depolarizing charges carried into the cell by the current (Figure 5).

The investigation pointed out that one of the two mechanisms $\left(\left[\mathrm{Ca}^{+2}\right]_{0}\right.$ modulation of DF) is not acting in CRN and KM (flat curves in Figure 5). Indeed in the $\mathrm{I}_{\mathrm{CaL}}$ formulations of these models, the DF is not dependent at all on $\left[\mathrm{Ca}^{+2}\right]_{\mathrm{o}}$. Of course this description is not realistic, and the right behavior of CRN and KM is actually relying on an incorrect assumption.

On the other hand, the EM model behaves completely the opposite of real cells (Figure 3). In silico analysis allowed to quantify the reasons: the $\left[\mathrm{Ca}^{+2}\right]_{0}$-induced modulation of $\mathrm{I}_{\mathrm{CaL}} \mathrm{DF}$ produces a large direct dependence of the current (see dashed line in Figure 5, here DF is the only $\left[\mathrm{Ca}^{+2}\right]_{\text {o }}$ modulated mechanism) while the CDI can induce only a modest inverse relationship (see dotted line in Figure 5, here CDI is the only $\left[\mathrm{Ca}^{+2}\right]_{\text {o modulated }}$ mechanism). When both mechanisms are in place the former prevails (Figure 5 continuous line) yielding the wrong behavior. The $\mathrm{I}_{\mathrm{NCX}}$ contribution further exacerbates this.

In conclusion, our results call for a new hAM able to reproduce correctly the $\left[\mathrm{Ca}^{+2}\right]_{\mathrm{o}}$ effect. Other models [11] have been recently proposed that deserve to be investigated in the future. Moreover, more experimental data are required in order to draw conclusions about the cardiac phenomena and not only on the models developed to describe them.

\section{References}

[1] Kirchhof P, Benussi S, Kotecha D, Ahlsson A, Atar D, Casadei B, et al. 2016 ESC Guidelines for the management of atrial fibrillation developed in collaboration with EACTS. Eur Heart J 2016;37:2893962. doi:10.1093/eurheartj/ehw210.

[2] Allessie M, Ausma J, Schotten U. Electrical, contractile and structural remodeling during atrial fibrillation. Cardiovasc Res 2002. doi:10.1016/S00086363(02)00258-4.

[3] Schotten U, Verheule S, Kirchhof P, Goette A. Pathophysiological mechanisms of atrial fibrillation: A translational appraisal. Physiol Rev 2011. doi:10.1152/physrev.00031.2009.

[4] Eisner DA, Diaz ME, Li Y, O'Neill SC, Trafford AW. Stability and instability of regulation of intracellular calcium. Exp Physiol 2005. doi:10.1113/expphysiol.2004.029231.

[5] Grandi E, Pandit S V., Voigt N, Workman AJ, Dobrev $\mathrm{D}$, Jalife J, et al. Human atrial action potential and $\mathrm{Ca}^{2+}$ model: Sinus rhythm and chronic atrial fibrillation. Circ Res 2011. doi:10.1161/CIRCRESAHA.111.253955.

[6] Koivumäki JT, Seemann G, Maleckar MM, Tavi P. In Silico screening of the key cellular Remodeling Targets in Chronic Atrial Fibrillation. PLoS Comput Biol 2014. doi:10.1371/journal.pcbi.1003620.

[7] Severi S, Corsi C, Cerbai E. From in vivo plasma composition to in vitro cardiac electrophysiology and in silico virtual heart: the extracellular calcium enigma. Philos Trans A Math Phys Eng Sci 2009;367:2203-23.

[8] Courtemanche M, Ramirez RJ, Nattel S. Ionic mechanisms underlying human atrial action potential properties: Insights from a mathematical model. Am J Physiol - Heart Circ Physiol 1998. doi:10.1152/ajpheart.1998.275.1.h301.

[9] Ellinwood N, Dobrev D, Morotti S, Grandi E. In silico assessment of efficacy and safety of Ikur inhibitors in chronic atrial fibrillation: Role of kinetics and statedependence of drug binding. Front Pharmacol 2017. doi:10.3389/fphar.2017.00799.

[10] Bébarová M. Advances in patch clamp technique: Towards higher quality and quantity. Gen Physiol Biophys 2012. doi:10.4149/gpb_2012_016.

[11] Colman MA, Saxena P, Kettlewell S, Workman AJ. Description of the human atrial action potential derived from a single, congruent data source: Novel computational models for integrated experimentalnumerical study of atrial arrhythmia mechanisms. Front Physiol 2018;9:1-17. doi:10.3389/fphys.2018.01211.

Address for correspondence:

Stefano Severi

Department of Electrical, Electronic

and Information Engineering,

University of Bologna,

Via dell'Università 50, 47522 Cesena (FC),

Italy

stefano.severi@unibo.it 